\title{
First experimental detection of antiproton in-flight annihilation on nuclei at $\sim 130 \mathrm{keV}$
}

\author{
H. Aghai-Khozani ${ }^{1,2}$, D. Barna ${ }^{3,4}$, M. Corradini ${ }^{5,6}$, R. Hayano ${ }^{4}$, M. Hori ${ }^{1,4}$, T. Kobayashi ${ }^{4}$, M. Leali ${ }^{5,6}$, \\ E. Lodi-Rizzini ${ }^{5,6, a}$, V. Mascagna ${ }^{5,6}$, M. Prest ${ }^{7,8}$, A. Soter ${ }^{1}$, K. Todoroki ${ }^{4}$, E. Vallazza ${ }^{9}$, L. Venturelli ${ }^{5,6}$, and \\ N. Zurlo 5,6 \\ 1 Max-Planck-Institut fur Quantenoptik, Hans-Kopfermann-Strasse 1, D-85748 Garching, Germany \\ 2 Physics Department, CERN, 1211 Geneva 23, Switzerland \\ 3 Wigner Institute for Particle and Nuclear Physics, H-1525 Budapest, Hungary \\ 4 Department of Physics, University of Tokyo, Tokyo 113-0033, Japan \\ ${ }_{5}^{5}$ Dipartimento di Chimica e Fisica per l'Ingegneria e per i Materiali, Università di Brescia, 25133 Brescia, Italy \\ 6 INFN, Gruppo Collegato di Brescia, 25133 Brescia, Italy \\ 7 Dipartimento di Scienze Fisiche e Matematiche, Università di Como, I-22100 Como, Italy \\ 8 INFN, Sezione di Milano Bicocca, I-20126 Milano, Italy \\ ${ }^{9}$ INFN, Sezione di Trieste, I-34127 Trieste, Italy
}

Received: 10 September 2012

Published online: 15 October 2012

(c) The Author(s) 2012. This article is published with open access at Springerlink.com

\begin{abstract}
The existing data of antinucleon-nucleon and antinucleon-nuclei annihilation cross-sections are confined to energies above about $1 \mathrm{MeV}$. Experimental limitations have prevented till now the lower energies data to be achieved in spite of the interest they represent for theoretical models. One of the unresolved question concerns the antiproton annihilation cross-section measured at LEAR on light nuclei in the MeV region, which show a saturation with the mass number of the target nucleus against any naive expectation. With regard to fundamental cosmology, the knowledge of the annihilation cross-sections at energies below $1 \mathrm{MeV}$ can contribute to understand the matter-antimatter asymmetry in the Universe. We present here the experimental demonstration of the feasibility of the measurement of antiproton-nuclei annihilation cross-sections in the $100 \mathrm{keV}$ region.
\end{abstract}

\section{Introduction}

We here report the first observation of in-flight antiproton-nucleus annihilations at an extremely low energy of 130 keV. The experiment was performed recently by the ASACUSA Collaboration [1] at the CERN's Antiproton Decelerator (AD), in which we measured antiproton $(\bar{p})$ annihilations on carbon, palladium and platinum targets. The experimental outcomes demonstrate that with the used technique the measurements of antiproton annihilation cross-section are feasible in the $100 \mathrm{keV}$ region.

At CERN's AD we have so far measured the $\bar{p}$-nucleus in-flight annihilation cross-sections for several targets, Mylar, $\mathrm{Ni}$, Sn, and Pt, at 5.3 MeV [2], and showed that the results are consistent with the predictions from the black-disk model with the Coulomb correction. In the MeV region there are also the data taken at LEAR but mostly with light targets [3-11]. These cross-sections deviate from the expected $Z A^{1 / 3}$ dependence [12] on the atomic charge $Z$ and mass number $A$ showing a saturation with $A$ [13-15], whereas at higher energies the $\bar{p}$ nucleus annihilation cross-section increases regularly with the number of nucleons in the nuclei: in the energy interval $20-200 \mathrm{MeV}$ the measurements performed at LEAR in the 1980's [16] show that the annihilation cross-sections are proportional to $A^{2 / 3}$. This last behavior is followed also by the antineutron data in the measured range $2-40 \mathrm{MeV}[17]$.

On the contrary when the $\bar{p}$ energy is below few $\mathrm{MeV}$ the $\bar{p} d$ and $\bar{p}{ }^{4} \mathrm{He}$ annihilation cross-sections values become even lower than the $\bar{p} p$ one. This is against any naive prediction of a scaling law with the number of nucleons in the target. This is confirmed also by the low-statistics data at about $2 \mathrm{MeV}[10,11]$ with Ne and ${ }^{3} \mathrm{He}$ targets. After the

\footnotetext{
${ }^{a}$ e-mail: lodi@bs.infn.it
} 


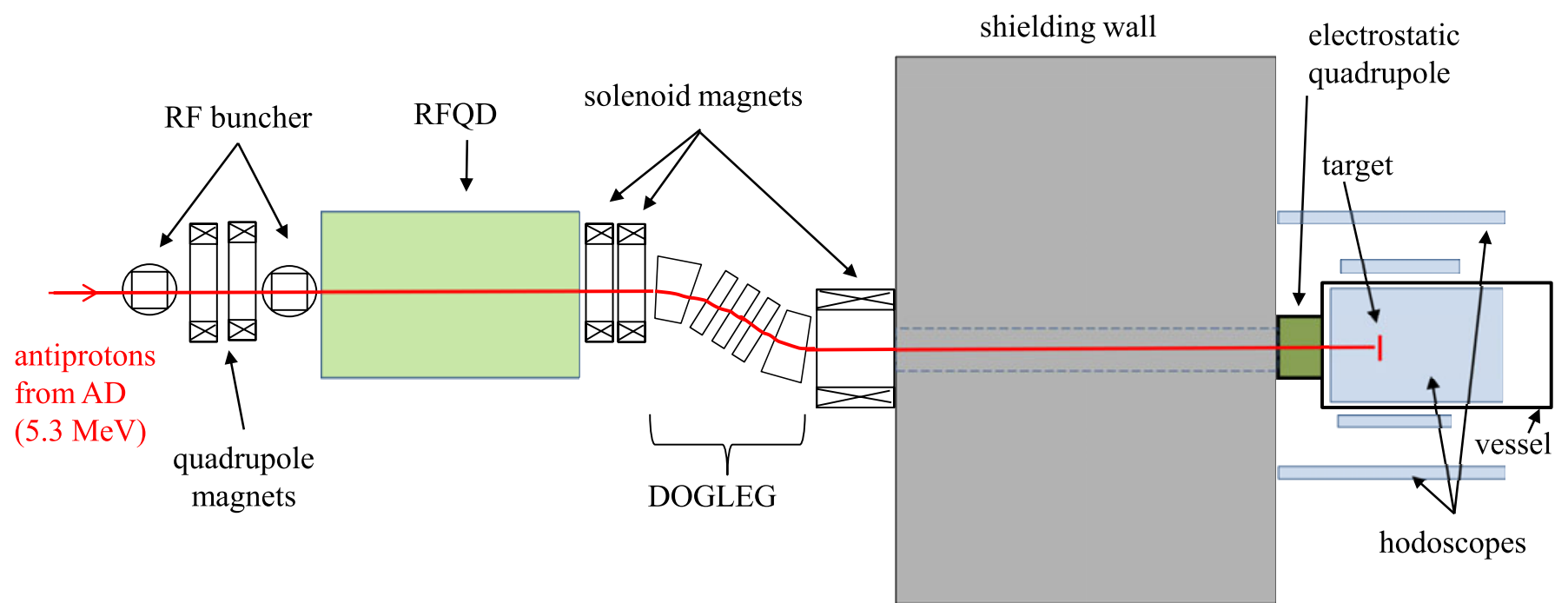

Fig. 1. A schematic view (not to scale) of the experimental setup used for the $\bar{p}$-nucleus annihilation cross-section measurements at $130 \mathrm{keV}$.

subtraction of the Coulomb contribution, the neon result has the same size as that of $p$, while the ${ }^{3}$ He result is twice as large as the others (including ${ }^{4} \mathrm{He}$ ) at the same energy.

When all of these cross-sections measurements together with the widths and the shifts of antiprotonic atoms [18-20] are considered inside several model analyses [13-15,21-27], a clear result emerges: the $\bar{p}$-nucleus interaction is largely dominated by important saturation effects, both in the negative energy bound state domain and in the positive energy reaction sector.

In addition both potential models $[15,23,27]$ and phenomenological analyses [28-30] state that the $\bar{N} N$ and $\bar{N}$ nucleus annihilation mechanisms occur in a thin region placed just outside the nuclear volume. As a consequence, the annihilation process has to be considered a valuable tool for probing the external region of the nucleus where for example the neutron/proton ratio or the extraction energy of the peripheral nucleons can be determined. Since more experimental inputs are needed to clarify the scenario, the possibility to go at lower energies, in the $100 \mathrm{keV}$ region, where no experimental data is available, is of interest.

The knowledge of the annihilations cross-sections for antinucleons, especially on light nuclei, is important also for the cosmology. In order to explain why the observable universe appears to be made almost entirely of matter, several mechanisms have been invoked. One possibility is that antimatter is distributed non-homogeneously in the Universe within the so-called "islands" of antimatter [31]. In the border region between matter and antimatter, the role of annihilation is important. Under specific conditions a relevant amount of annihilations is expected to be occured at the nucleosynthesis time before the recombination time with energies between $1 \mathrm{eV}$ and $1 \mathrm{MeV}$. Since the values of the annihilation cross-sections affect strongly the evolution of the involved processes, the measurements of the antinucleon annihilation cross-sections in the $\mathrm{eV}-\mathrm{MeV}$ region are expected.

\section{Experimental setup}

\subsection{Principle}

In the present experiment we decelerated the $\bar{p}$ s from $5.3 \mathrm{MeV}$ to $130 \mathrm{keV}$ by means of the radiofrequency quadrupole decelerator (RFQD) [32] and we used a beam line, a vessel with solid targets and detectors specifically designed for this experiment, as depicted in fig. 1. Measuring the annihilation cross-sections at this low energy and with a pulsed beam is non-trivial, and requires the development of a non-conventional scheme. The key measurement principles are here enumerated:

1) The RFQD delivers a pulsed beam of $\bar{p}$. Only a fraction (about $20 \%$ ) of the antiprotons from $\mathrm{AD}\left(3 \times 10^{7}\right.$ every bunch repeated every $\sim 100 \mathrm{~s}$ ) is decelerated to $130 \mathrm{keV}$, while a similar number reaches the exit of RFQD at $5.3 \mathrm{MeV}$. We installed a magnetic spectrometer "DOGLEG" to transport the decelerated $\bar{p}$ s and we built a shielding wall comprising $0.8 \mathrm{~m}$ thick iron and $2 \mathrm{~m}$ thick concrete between the DOGLEG and the target chamber in order to suppress background caused by stray $\bar{p}$ annihilations.

2) The $\bar{p}$ beam decelerated by the RFQD has a large emittance of $\sim 100 \pi \mathrm{mm} \cdot \mathrm{mrad}$ and its halo can be pernicious for the experiment. In order to ensure that the $\bar{p}$ beam is focussed on the target, and do not hit the target frame $(8 \mathrm{~cm}$ 


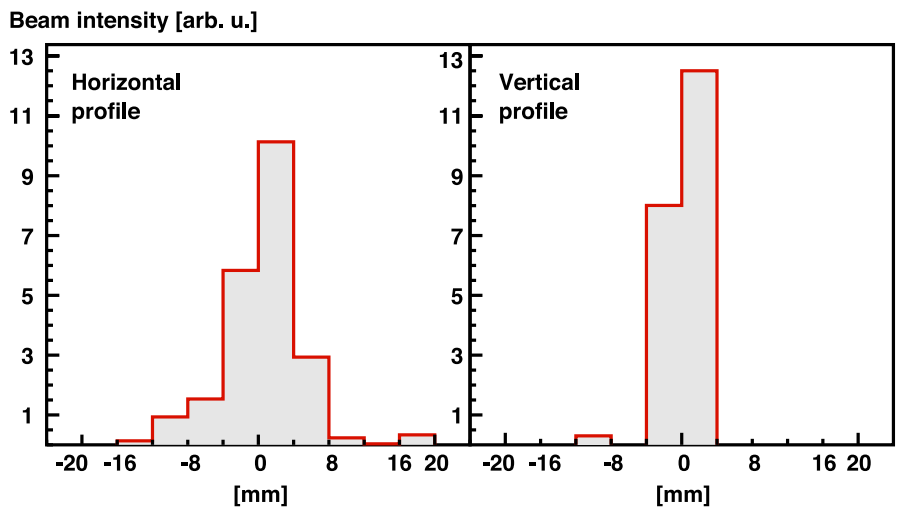

Fig. 2. The $\bar{p}$ beam profile $(x, y)$ on the target, $z$ being around $67 \mathrm{~cm}$ from the entrance of the target vessel.

Table 1. The targets used in the present experiment. For the definition of equivalent thickness, see main text. The thicknesses shown here are nominal.

\begin{tabular}{lrlrr}
\hline Target & $(A, Z)$ & Description & Equivalent thickness (nm) & Relative Rutherford \\
\hline Empty & & $8 \mathrm{~cm}$ diameter frame only & \\
$\mathrm{C}$ & $(12,6)$ & $\sim 70 \mathrm{~nm}$ carbon foil fixed to the frame & 70 & 1 \\
$\mathrm{Pd}$ & $(106,46)$ & $\sim 19 \mathrm{~nm}$ Pd sputtered on the $\sim 70 \mathrm{~nm}$ carbon foil & $130+70$ & $16+1$ \\
$\mathrm{Pt}$ & $(195,78)$ & $\sim 5 \mathrm{~nm}$ Pt sputtered on the $\sim 70 \mathrm{~nm}$ carbon foil & $70+70$ & $12+1$ \\
\hline
\end{tabular}

diameter), we installed an aperture of $1 \mathrm{~cm}$ in diameter in the middle of the DOGLEG, and a variable-aperture iris $(1 \div 13 \mathrm{~cm}$ in diameter) at the exit of the DOGLEG.

3) To focus the $\bar{p}$ s on the target, placed $67 \mathrm{~cm}$ downstream of the target-vessel entrance (i.e., some $3 \mathrm{~m}$ downstream of the DOGLEG), we developed and installed an electrostatic quadrupole [33] between the DOGLEG and the target vessel (see fig. 1). The alignment and focussing of the beam was checked by a retractable beam profile monitor [34] based on the secondary electron emission, with an active area of $40 \times 40 \mathrm{~mm}^{2}$ and a spatial resolution of $4 \mathrm{~mm}$ (see fig. 2).

4) A very small fraction of the $\bar{p}$ s annihilate on the target (whose number we want to count), while the rest travel to the end of the target vessel and annihilate. In order to unambiguously identify the annihilations on the target, we rely on the time separation. This can be achieved with a short pulse length and a relative long target vessel. The pulse length was reduced from the original 150-200 ns value to about $70 \mathrm{~ns}$ by using an electronic chopper inserted inside the DOGLEG. According to the detector time resolution (some ns), the vessel length of $170 \mathrm{~cm}$ was chosen.

5) Some $\bar{p}$ s also undergo Rutherford scattering on the target with a probability proportional to $Z^{2} d$, where $Z$ is the atomic number of the target, and $d$ is the target thickness. Rutherford-scattered $\bar{p}$ seach the lateral wall of the vessel and annihilate. In order to have a clean separation of those annihilations from the signal events, the vessel diameter of $120 \mathrm{~cm}$ was chosen.

6) The $\bar{p}$ s Rutherford-scattered at $90^{\circ}$ can move within the target foil, slow down, stop (the range of $130 \mathrm{keV} \bar{p} \mathrm{~s}$ is less than few $\mu \mathrm{m}$ in solids) and annihilate. Since it is impossible to tell such annihilations from the in-flight events based on the timing, it is important to use thin targets. Rutherford scattering and energy-loss simulations have shown that the probability of at-rest $\bar{p}$ annihilations is less than that of the in-flight annihilations for all the targets we used.

\subsection{Targets}

The targets used in the present experiment are listed in table 1 . The target frame size, $8 \mathrm{~cm}$, was chosen to minimize the $\bar{p}$ annihilations on the frame from beam halos. This large size in turn sets a lower limit to the target thickness to be in the range of 70-100 nm. The Pd and Pt targets were prepared at Technische Universität München by sputtering the $\mathrm{Pd} / \mathrm{Pt}$ on a carbon foil. The thicknesses of metals sputtered on the carbon foil were chosen such that the in-flight nuclear annihilation rates do not depend strongly on the element. This is indicated by the equivalent carbon thicknesses, calculated by assuming the Coulomb-modified "black-disk" model of ref. [15]; in the case of Pt, for example, Pt and the carbon backing contribute equally to the in-flight annihilations.

The last column is the relative Rutherford scattering probability (normalized to the $70 \mathrm{~nm} \mathrm{C}$ ), showing that the Rutherford scattering events from $\mathrm{Pd}$ and $\mathrm{Pt}$ are an order of magnitude more frequent than from the $\mathrm{C}$ target. 


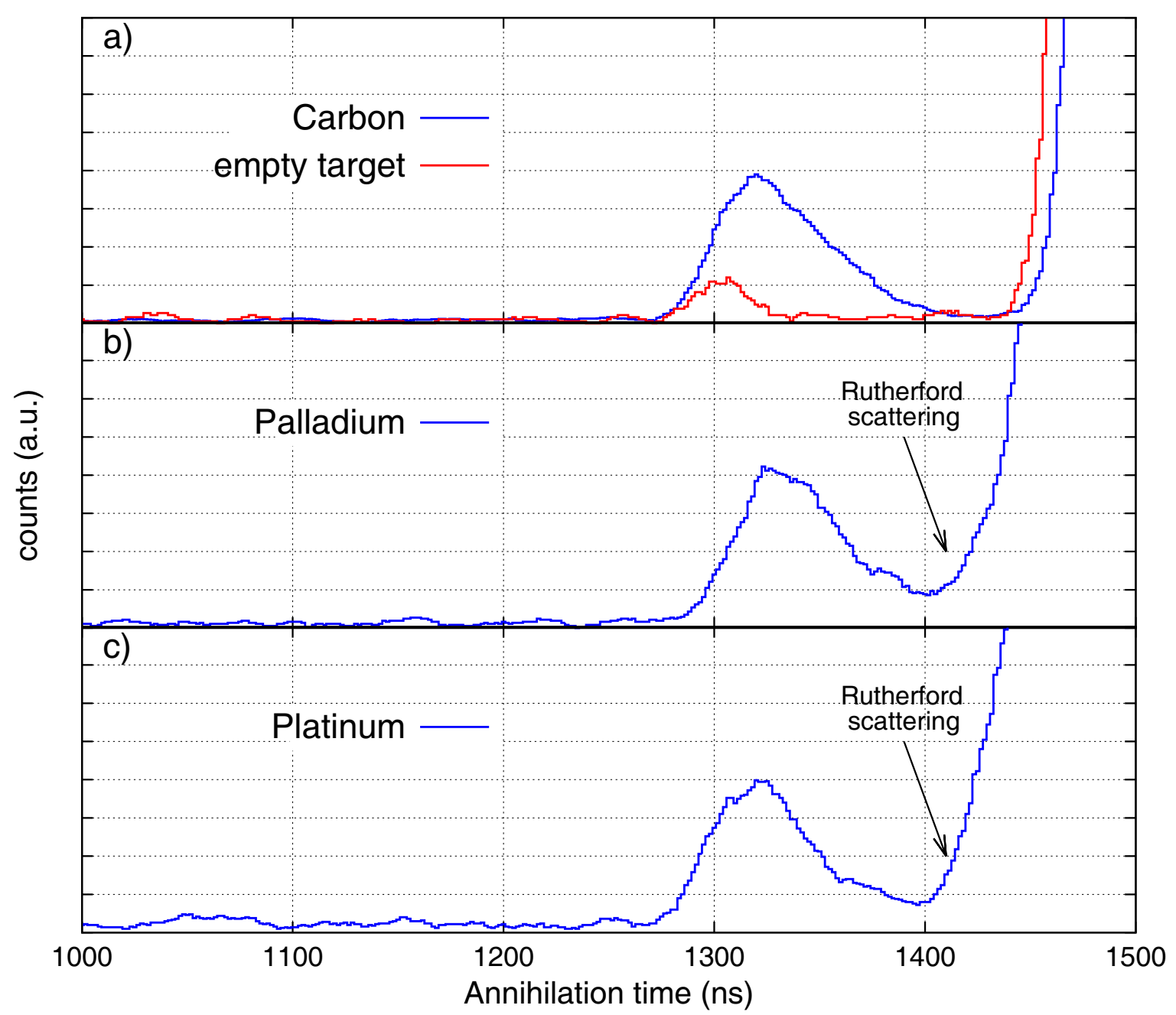

Fig. 3. The $\bar{p}$-annihilation time distributions for a) $\mathrm{C}$ target (blue line) and empty target (red line), b) $\mathrm{Pd}$ target and c) $\mathrm{Pt}$ target.

The vessel, evacuated to $\sim 10^{-7} \mathrm{mb}$ and connected directly to the RFQD (and hence to the AD ring) with no material window along the antiprotons path in order to avoid a possible source of background annihilations, was designed so that one of the four targets (empty, C, Pd and Pt), normally in the off-axis parking position, could be rotated into the beam without breaking the vacuum. The beam-profile monitor was attached to the head of an on-axis rod, and could be moved to the target position, or retracted to the end wall.

\subsection{Detectors}

The $\bar{p}$ annihilations were detected with $\sim 600$ fast scintillator bars provided by FNAL arranged in planar hodoscopes, placed on top, bottom, left and right of the target vessel (see fig. 1). Of these, $\sim 500$ were readout by multi-anode PMTs [35], and the remaining $~ 100$ were readout by MPPCs [36]. Both the PMTs and the MPPCs were readout with dedicated frontend boards hosting suitable ASICs and Altera FPGAs; the boards are handled by a VME electronics designed for the purpose. The analog signals of the multianode PMTs are processed by the ASICs with a sample and hold circuit while the full wave forms of the MPPCs are digitized and stored. In total, these detectors cover about $\sim 20 \%$ of the solid angle.

In the present paper, we show the results from one of the modules placed below the target vessel, to demonstrate that the in-flight annihilations on the target can be well identified simply by the timing cut. More detailed analyses including vertex analyses will be presented in a forthcoming extended report.

In addition, a Čherenkov detector [37], known to have good linearity, was placed below the target vessel and its wave form was recorded by a digital oscilloscope, in order to monitor the $\bar{p}$ beam intensity. 


\section{Results}

In fig. 3 we present the $\bar{p}$-annihilation time distributions measured with one of the fast scintillator modules $\left(1 \times 0.45 \mathrm{~m}^{2}\right.$, 31 scintillator bars) placed below the target vessel during some hours of data taking per sample; from top to bottom, a) C target with the so-called "empty target" (only the target frame), b) Pd target and c) Pt target.

These spectra show the following components: 1) a nearly constant background due to the $\pi$ - $\mu$-e decays caused by the annihilations of stray $\bar{p} \mathrm{~s}, 2) \bar{p}$ annihilations on the target, 3) annihilations of Rutherford-scattered $\bar{p} \mathrm{~s}$ on the lateral wall of the target vessel, and 4) annihilations of $\bar{p}$ s which reached the end wall of the vessel.

As shown, the annihilations on the target are clearly visible in the interval $\Delta t=1280-1400$ ns, well separated in time from other components, while there are very few counts in the case of empty-target run (about 5-10\% of the counts with the targets), indicating that the $\bar{p}$ beam is well centered and focused on the target and is not hitting the $8 \mathrm{~cm}$ diameter target frame. The $\pi$ - $\mu$-e background is almost completely suppressed by the shielding placed upstream of the target vessel as shown before the $\Delta t$ interval. The Rutherford-scattering component on the lateral wall of the vessel, which is significant in the case of $\mathrm{Pt}$ and $\mathrm{Pd}$ targets while it is much less pronounced for the $\mathrm{C}$ target, does not appreciably contaminate the $\Delta t$ region of the annihilation events on the target. The dominant end wall contribution appears later in time, and hence poses no problem to our measurement.

\section{Conclusions}

This is the first time that $\bar{p}$-nucleus in-flight annihilations were observed in the extremely low energy range of $\sim 100 \mathrm{keV}$. The described experimental method permits to perform measurements of the cross-section values for antiproton of $100 \mathrm{keV}$ energy. In our forthcoming full report, we will use all the detector channels, determine the target thicknesses by the Rutherford-backscattering method, and report the absolute annihilation cross-sections.

Recently, the construction of a new $\bar{p}$ decelerator ring called ELENA was approved at CERN [38]. This ring will decelerate the $5.3 \mathrm{MeV} \bar{p}$ s from the $\mathrm{AD}$ ring down to $100 \mathrm{keV}$ with electron cooling, the design emittance being $5 \pi \mathrm{mm} \cdot \mathrm{mrad}$, so that the $\bar{p}$ beam can be focussed on a smaller spot of $\lesssim 1 \mathrm{~cm}$ in diameter. This will make it possible, among other things, to measure $\bar{p}$ annihilations on thinner $(\lesssim 50 \mathrm{~nm})$ targets. The method established by the present experiment paves the road toward such future measurements which can contribute to understand the antinucleon-nucleon and antinucleon-nucleus annihilation mechanism and to shed light on the unresolved problem of the saturation of the cross-section whith the mass number of the nucleus. The knowledge of the annihilation crosssections at very low energy is also important to correctly determine the properties of matter-antimatter interaction as requested by several models attempting to explain the matter/antimatter asymmetry in the Universe. In addition, the successful commissioning of the electrostatic quadrupole, one of the key devices for the ELENA user beamlines (which will use electrostatic bending and focussing elements), is an important step towards the ELENA beamline construction.

This work was supported by Istituto Nazionale di Fisica Nucleare (INFN), Università di Brescia, MEXT Japan (grant no. 20002003), the European Young Investigator Awards (EURYI) of the European Science Foundation and the Deutsche Forschungsgemeinschaft (DFG), and the Munich Advanced Photonics (MAP) cluster of DFG. We thank the CERN Antiproton Decelerator and Proton Synchrotron operational staff. We also thank Dominik Seiler for the preparation of the targets.

Open Access This is an open access article distributed under the terms of the Creative Commons Attribution License (http://creativecommons.org/licenses/by/3.0), which permits unrestricted use, distribution, and reproduction in any medium, provided the original work is properly cited.

\section{References}

1. R.S. Hayano et al., CERN-SPSC-2005-002, SPSC-97-19-ADD-2.

2. A. Bianconi et al., Phys. Lett. B 704, 461 (2011).

3. M. Agnello et al., Phys. Lett. B 256, 349 (1991).

4. A. Bertin et al., Phys. Lett. B 369, 77 (1996).

5. A. Benedettini et al., Nucl. Phys. B 56, 55 (1997).

6. A. Bertin et al., Phys. Lett. B 414, 220 (1997).

7. A. Zenoni et al., Phys. Lett. B 461, 413 (1999).

8. A. Zenoni et al., Phys. Lett. B 461, 405 (1999).

9. A. Zenoni et al., Nucl. Instrum. Methods B 447, 512 (2000).

10. A. Bianconi et al., Phys. Lett. B 481, 194 (2000). 
11. A. Bianconi et al., Phys. Lett. B 492, 254 (2000).

12. L.D. Landau, E.M. Lifshitz, Course of Theoretical Physics - Quantum Mechanics (Non-Relativistic Theory), Vol. 3 (Butterworth-Heinemann, 1981).

13. A. Bianconi, G. Bonomi, M.P. Bussa, E. Lodi Rizzini, L. Venturelli, Phys. Lett. B 483, 353 (2000).

14. A. Bianconi, G. Bonomi, E. Lodi Rizzini, L. Venturelli, Phys. Rev. C 62, 014611 (2000).

15. C.J. Batty, E. Friedman, A. Gal, Nucl. Phys. A 689, 721 (2001).

16. G. Bendiscioli, D. Kharzeev, Riv. Nuovo Cimento 17, 1 (1994).

17. F. Iazzi et al., Phys. Lett. B 475, 378 (2000).

18. M. Augsburger et al., Phys. Lett. B 461, 317 (1999).

19. S. Wycech et al., Nucl. Phys. A 655, 257c (1999) Harmand et al., ibidem.

20. R. Schmidt et al., Phys. Rev. C 60, 0543309 (1999).

21. S.Wycech, A.M. Green, J.A. Niskanen, Phys. Lett. B 152, 308 (1985).

22. G.Q. Liu, J.M. Richard, S. Wycech, Phys. Lett. B 260, 15 (1991).

23. Ye.S. Golubeva, L.A. Kondratyuk, Nucl. Phys. B 56A, 103 (1997).

24. E. Friedman, A. Gal, Phys. Lett. B 459, 43 (1999).

25. E. Friedman, A. Gal, Nucl. Phys. A 658, 345 (1999).

26. K.V. Protasov, G. Bonomi, E. Lodi Rizzini, A. Zenoni, Eur. Phys. J. A 7, 429 (2000).

27. V.A. Karmanov, K.V. Protasov, A.Yu. Voronin, Eur. Phys. J. A 8, 429 (2000).

28. W. Brückner et al., Z. Phys. A 339, 379 (1991).

29. J. Haidembauer, T. Hippchen, K. Holinde, J. Speth, Z. Phys. A 334, 467 (1989).

30. A.S. Jensen, in Antiproton-nucleon and antiproton-nucleus interactions, edited by F. Bradamante, J.M. Richard, R. Klapish, Ettore Majorana international science series (Plenum Press 1990) p. 205.

31. A.G. Cohen, A. de Rújula, S.L. Glashow, Astrophys. J. 495, 539 (1998).

32. A.M. Lombardi, W. Pirkl, Y. Bylinsky, in 2001 Particle Physics Accelerator Conference (Chicago, IL), edited by P. Lucasa, S. Webber (IEEE, Piscataway, 2001) pp. 585-587.

33. M. Hori et al., in preparation.

34. K. Todoroki, M. Hori, JINST 7, C02052 (2012).

35. M. Corradini et al., in preparation.

36. A. Soter et al., in preparation.

37. M. Hori et al., Nucl. Instrum. Methods A 496, 102 (2003).

38. W. Oelert et al., CERN-SPSC-2009-026, SPSC-P-338. 